\title{
A NOTE ON INFINITE-DIMENSIONAL SPACES DEFINED BY TOPOLOGICAL GAMES
}

\author{
YASUNAO HATTORI
}

\begin{abstract}
We get the following result: For a totally normal space $X, X$ is a strongly countable dimensional space if and only if $X$ is an $\mathbf{F}(\mathbf{d i m})$-like space. This result answers a question of $\mathrm{R}$. Telgársky.
\end{abstract}

Let $\mathbf{K}$ denote a class of spaces which are hereditary with respect to closed subspaces and $\sigma \mathbf{K}$ a class of all spaces which are countable unions of closed subspaces belonging to K. Recall from [T] that a space is called K-like if Player I has a winning strategy in $G(\mathbf{K}, X)$. In [T, Question 4.10], R. Telgársky asked whether $X$ is $\mathbf{K}$-like if it is $\sigma \mathbf{K}$-like. Under the assumption of $\mathrm{CH}$, he gave a negative answer to this question (see [ $\mathbf{Y}_{1}$, Example 5.1]). He used the Lusin set to construct the example. However K. Kunen [K] showed that $\mathrm{MA}+{ }_{\neg} \mathrm{CH}$ implies that there are no (useful) Lusin sets. Hence the assumption of $\mathrm{CH}$ seems to be essential to the construction of the R. Telgársky's example. In this note, we answer the above question without any set-theoretic assumption beyond ZFC. Let $\mathbf{F}(\mathbf{d i m})$ denote the class of all finitedimensional spaces (in the sense of the covering dimension $\operatorname{dim}$ ) and $N$ the set of all natural numbers. For each $n, i, k \in N$ and $\alpha=\left\langle k_{1}, \ldots, k_{n}\right\rangle \in N^{n}$, let

$$
\alpha_{-i}=\left\langle k_{1}, \ldots, k_{n-i}\right\rangle, \quad \alpha_{-n}=\varnothing \quad \text { and } \alpha \oplus k=\left\langle k_{1}, \ldots, k_{n}, k\right\rangle .
$$

For each admissible sequence $T=\left\langle E_{0}, E_{1}, \ldots, E_{2 n}\right\rangle$ in $G(\mathbf{K}, X)$ and a closed set $F$ of $X$ with $F \cap s(T)=\varnothing$, let $\langle T ; s(T) ; F\rangle$ denote an admissible sequence $\left\langle E_{0}, E_{1}, \ldots, E_{2 n}, s(T), F\right\rangle$ in $G(\mathbf{K}, X)$, where $s$ is a strategy of Player I. We refer the readers to $[\mathbf{T}]$ for the descriptions and details of the topological games and to $[\mathbf{P}]$ for the dimension theory.

To answer the above question, we need the following result which is of interest in itself.

THEOREM. Let $X$ be a totally normal space. Then $X$ is strongly countable dimensional (i.e. $X \in \sigma \mathbf{F}(\mathbf{d i m})$ ) if and only if $X$ is $\mathbf{F ( d i m ) - l i k e . ~}$

Proof. Let $X$ be an $\mathbf{F}$ (dim)-like space and $s$ a winning strategy of Player $I$ in $G(\mathbf{F}(\mathbf{d i m}), X)$. Without loss of generality we can assume that $X$ is infinite-dimensional. Let $T(\langle\varnothing, \varnothing\rangle)=\left\{\left\langle E_{0}\right\rangle\right\}$ and $Z(\langle\varnothing, \varnothing\rangle)=s\left(E_{0}\right)$, where $E_{0}=X$. Since $X$

Received by the editors March 7, 1983 and, in revised form, July 24, 1984.

1980 Mathematics Subject Classification. Primary 54F45; Secondary 54D15.

Key words and phrases. Topological game, covering dimension, strongly countable dimension, totally normal space. 
is totally normal, for each $n \in N$ and $\langle\alpha, \beta\rangle \in N^{n} \times N^{n}$, we can inductively construct families $\mathscr{S}(T,\langle\alpha, \beta\rangle)$ of closed subsets of $X$ for each $T \in \mathscr{T}\left(\left\langle\alpha_{-1}, \beta_{-1}\right\rangle\right)$, families $\mathscr{T}(\langle\alpha, \beta\rangle)$ of admissible sequences in the game $G(\mathbf{F}(\mathbf{d i m}), X)$ and subsets $Z(\langle\alpha, \beta\rangle)$ of $X$ which satisfy the following conditions:

$$
\mathscr{T}(\langle\alpha, \beta\rangle)=\left\{\begin{array}{l}
\left\{\langle T ; s(T) ; F\rangle: T \in \mathscr{T}\left(\left\langle\alpha_{-1}, \beta_{-1}\right\rangle\right) \text { and } F \in \mathscr{S}(T,\langle\alpha, \beta\rangle)\right\}, \\
\text { if } \mathscr{T}\left(\left\langle\alpha_{-1}, \beta_{-1}\right\rangle\right) \neq \varnothing \text { and } \mathscr{S}(T,\langle\alpha, \beta\rangle) \neq \varnothing, \\
\varnothing, \quad \text { otherwise. }
\end{array}\right.
$$

$$
\begin{aligned}
& \mathscr{S}(T,\langle\alpha, \beta\rangle) \text { is locally finite at each point of } E_{2 n-2}-s(T), \\
& \text { where } T=\left\langle E_{0}, \ldots, E_{2 n-2}\right\rangle . \\
& \bigcup\left\{\mathscr{S}\left(T,\left\langle\alpha_{-1} \oplus k, \beta_{-1} \oplus m\right\rangle\right): k, m \in N\right\} \text { is a cover of } \\
& E_{2 n-2}-s(T), \text { where } T=\left\langle E_{0}, \ldots, E_{2 n-2}\right\rangle .
\end{aligned}
$$

Now, for each $\langle\alpha, \beta\rangle \in N^{n} \times N^{n}$ and $n \in N$, put

$$
\mathscr{S}(\langle\alpha, \beta\rangle)=\bigcup\left\{\mathscr{S}(T ;\langle\alpha, \beta\rangle): T \in \mathscr{T}\left(\left\langle\alpha_{-1}, \beta_{-1}\right\rangle\right)\right\} .
$$

Claim 1. $\mathscr{S}(\langle\alpha, \beta\rangle)$ is locally finite at each point of $X-Z\left(\left\langle\alpha_{-1}, \beta_{-1}\right\rangle\right)$.

Proof. Let $x \in X-Z\left(\left\langle\alpha_{-1}, \beta_{-1}\right\rangle\right)$. Without loss of generality we can assume that $\langle\alpha, \beta\rangle \in N^{n} \times N^{n}$ and $n \geqslant 2$. Since $x \notin Z\left(\left\langle\alpha_{-1}, \beta_{-1}\right\rangle\right) \supset Z\left(\left\langle\alpha_{-2}, \beta_{-2}\right\rangle\right)$, there is a neighbourhood $U$ of $x$ which meets at most finitely many members of $\mathscr{S}\left(\left\langle\alpha_{-1}, \beta_{-1}\right\rangle\right)$, by the inductive assumption. Let $F \in \mathscr{S}\left(T,\left\langle\alpha_{-1}, \beta_{-1}\right\rangle\right)$ with $F \cap U \neq \varnothing$, where $T=\left\langle E_{0}, \ldots, E_{2 n-4}\right\rangle \in \mathscr{T}\left(\left\langle\alpha_{-2}, \beta_{-2}\right\rangle\right)$. By the condition (1), $\mathscr{S}\left(T^{\prime},\left\langle\alpha_{-1}, \beta_{-1}\right\rangle\right)$ is locally finite in $F-s\left(T^{\prime}\right)$, where $T^{\prime}=\langle T ; s(T) ; F\rangle$ and hence so in $X-s\left(T^{\prime}\right)$. By the conditions (3) and (5), it follows that $\mathscr{S}\left(T^{\prime},\left\langle\alpha_{-1}, \beta_{-1}\right\rangle\right)$ is locally finite at each point of $X-Z\left(\left\langle\alpha_{-1}, \beta_{-1}\right\rangle\right)$. Let $U(F)$ be a neighbourhood of $x$ which meets at most finitely many members of $\mathscr{S}\left(T^{\prime},\left\langle\alpha_{-1}, \beta_{-1}\right\rangle\right)$. Put

$$
V=U \cap\left(\bigcap\left\{U(F): F \in \mathscr{S}\left(\left\langle\alpha_{-1}, \beta_{-1}\right\rangle\right) \text { and } U \cap F \neq \varnothing\right\}\right) .
$$

It is clear that $V$ is a neighbourhood of $x$ which meets at most finitely many members of $\mathscr{S}(\langle\alpha, \beta\rangle)$.

Claim 2. $Z(\langle\alpha, \beta\rangle)$ is a closed finite-dimensional subspace of $X$.

Proof. We shall prove by induction. It is obvious that $Z(\langle\varnothing, \varnothing\rangle)$ is a closed finite-dimensional subspace of $X$. Let $n \in N$ and $\langle\alpha, \beta\rangle \in N^{n} \times N^{n}$. If $\mathscr{T}(\langle\alpha, \beta\rangle)$ $=\varnothing$, then $Z(\langle\alpha, \beta\rangle)=Z\left(\left\langle\alpha_{-1}, \beta_{-1}\right\rangle\right)$. Assume that $\mathscr{T}(\langle\alpha, \beta\rangle) \neq \varnothing$. From Claim 1, it is easy to see that $Z(\langle\alpha, \beta\rangle)$ is a closed subset of $X$. Now, let $\beta=\beta_{-1} \oplus m$ and $F$ be a closed subset of $Z(\langle\alpha, \beta\rangle)$ with $F \cap Z\left(\left\langle\alpha_{-1}, \beta_{-1}\right\rangle\right)=\varnothing$. From Claim 1 and the condition (5), $\{s(T) \cap F: T \in \mathscr{T}(\langle\alpha, \beta\rangle)\}$ is a locally finite closed cover of $F$. For each $T \in \mathscr{T}(\langle\alpha, \beta\rangle), \operatorname{dim} s(T) \leqslant m$ by the condition (4). Thus $\operatorname{dim} s(T) \cap F \leqslant m$ 
and hence $\operatorname{dim} F \leqslant m$. Hence $\operatorname{dim} Z(\langle\alpha, \beta\rangle) \leqslant \max \left\{\operatorname{dim} Z\left(\left\langle\alpha_{-1}, \beta_{-1}\right\rangle\right), m\right\}<\infty$ by the inductive assumption. Put

$$
Z=Z(\langle\varnothing, \varnothing\rangle) \cup\left(\bigcup\left\{Z(\langle\alpha, \beta\rangle):\langle\alpha, \beta\rangle \in N^{n} \times N^{n}, n \in N\right\}\right) .
$$

To complete the proof of the "if" part it is enough to verify that $X=Z$. Assume that $x_{0} \in X-Z$. Since $x_{0} \notin Z(\langle\varnothing, \varnothing\rangle)=s\left(E_{0}\right)$, there are $k_{1}, m_{1} \in N$ and $E_{2} \in$ $\mathscr{S}\left(\left\langle E_{0}\right\rangle,\left\langle\left\langle k_{1}\right\rangle,\left\langle m_{1}\right\rangle\right\rangle\right)$ such that $x_{0} \in E_{2}$ by the condition (2). Put $E_{1}=s\left(E_{0}\right)$. Since $\left\langle E_{0}, E_{1}, E_{2}\right\rangle \in \mathscr{T}\left(\left\langle k_{1}\right\rangle,\left\langle m_{1}\right\rangle\right)$, it follows that $E_{3} \subset Z\left(\left\langle k_{1}\right\rangle,\left\langle m_{1}\right\rangle\right)$, where $E_{3}=s\left(E_{0}, E_{1}, E_{2}\right)$. Hence $x_{0} \in E_{2}-E_{3}$. Continuing in this manner we get a play $\left\langle E_{0}, E_{1}, \ldots\right\rangle$ of $G(\mathbf{F}(\mathrm{dim}), X)$ such that $E_{1}=s\left(E_{0}\right), E_{2 n+1}=s\left(E_{0}, E_{1}, \ldots E_{2 n}\right)$ for each $n \in N$ and $x_{0} \in \bigcap\left\{E_{2 n}: n \in N\right\}$. This is a contradiction. The "only if" part is obvious. The proof is complete.

The following example answers [T, Question 4.10] negatively.

EXAMPLE. There is a hereditarily paracompact $\sigma \mathbf{F}(\mathrm{dim})$-like space which is not F(dim)-like.

Proof. Let $K_{\omega}$ denote the subspace of the Hilbert cube $I^{\omega}$ consisting of all points in $I^{\omega}$ which have only finitely many nonzero coordinates. Let $X$ be a space $\left(I^{\omega}\right)_{K_{\omega}}$ defined as the set $I^{\omega}$ with the new topology generated by the base consisting of all sets of the form $U \cup M$, where $U$ is an open set of the Hilbert cube $I^{\omega}$ and $M \subset I^{\omega}-K_{\omega}$. Since $K_{\omega}$ is a strongly countable dimensional closed subspace of $X$ and $\operatorname{dim} X-K_{\omega}=0, X$ is a hereditarily paracompact $\sigma \mathbf{F}($ dim)-like space. But $X$ is not a strongly countable dimensional space (see [EP, Example 3.4]). Hence $X$ is not F(dim)-like by the above theorem. This completes the proof.

REMARKS. 1. We can replace the assumption "totally normal" by "normal and hereditarily subparacompact" in the above theorem. This proof is quite parallel to the above one.

2. Recently $Y$. Yajima gave a positive partial answer to the above question [ $\mathbf{Y}_{2}$, Theorem 2.1]: Let $X$ be a $P$-space (in the sense of Morita [M]). Then $X$ is K-like if $X$ is $\sigma \mathrm{K}$-like.

\section{REFERENCES}

[EP] R. Engelking and E. Pol, Countable-dimensional spaces: A survey, Dissertationes Math. 216 (1983).

[K] K. Kunen, Lusin spaces, Topology Proc. 1 (1976), 191-199.

[M] K. Morita, Products of normal spaces with metric spaces, Math. Ann. 154 (1964), 365-382.

[P] A. R. Pears, Dimension theory of general spaces, Cambridge Univ. Press, Cambridge, 1975.

[T] R. Telgársky, Spaces defined by topological games, Fund. Math. 88 (1975), 193-223.

[Y, Y. Yajima, Topological games and products. III, Fund. Math. 117 (1983), 223-238.

$\left[\mathrm{Y}_{2}\right] \ldots$, Notes on topological games, Fund. Math. 121 (1984), 31-40.

Department of Mathematics, Osaka Kyoiku University, Tennoji, Osaka, 543, Japan 